\title{
AN INTEGRATED APPROACH TO AGRICULTURAL CROP CLASSIFICATION USING SPOT5 HRV IMAGES
}

\author{
Chang $\mathrm{Yi}^{1,2, *}$, Yaozhong Pan ${ }^{1,2}$, Jinshui Zhang ${ }^{1,2}$ \\ ${ }^{1}$ College of Resources Science and Technology, Beijing Normal University, Beijing, China, \\ 100875 \\ ${ }^{2}$ State key Laboratory of Earth Surface Processes and Resource Ecology System (Beijing \\ Normal University), Beijing, China, 100875 \\ * Corresponding author, Address: 05 Shuo, College of Resources Science and Technology, \\ Beijing Normal University, 19 Xinjiekouwai Street, Beijing, 100875, P. R. China, Tel: +86- \\ 13581929372, Email: yichang531@ires.cn
}

Abstract: An integrated method that incorporates the advantages of per-parcel and perpixel approaches as well as spectral and spatial characteristics was proposed for crop classification of a typical agricultural area in south-east China using SPOT5 HRV data. The co-occurrence texture was employed to evaluate the heterogeneity of the image data. The average parcel textures determined each parcel defined by the crop boundaries to be classified whether on a per-parcel or per-pixel basis. The optimal threshold in the span of texture ranges was detected by trend analysis, which assigned the proportions of each approach in the integration, thus to produce the best integrated classification. It was suggested that this integrated approach can be effectively implemented to produce crop classification maps with higher accuracy from satellite images of medium and high spatial resolution in a complex agricultural environment, where both homogeneous and heterogeneous crop fields occur side by side.

Keywords: $\quad$ per-parcel, per-pixel, remote sensing, agricultural crop classification, SPOT5

\section{INTRODUCTION}

In recent years, advances in satellite imaging technology have boosted multiple spatial applications in which land cover information is an essential 
prerequisite (Lo and Choi, 2004). For the agricultural application, it requires a quantitative processing of digital images with high accuracy and reliability.

One crucial technique of crop mapping from remotely sensed data is the automated image classification, which usually operates on a per-pixel basis to categorize pixels separately into one of the pre-determined classes according to their spectral characteristics. However, as for agricultural applications, groups of pixels that represent the same crop type may not necessarily have the same spectral information due to the variation in soil moisture conditions, nutrient limitations or pests and diseases (De Wit and Clevers, 2004). Also, boundaries that cross pixels are an additional problem, as the spectral information of the pixel is then a combination of the reflectance from two or more land cover types (Smith and Fuller, 2001). Thus, doubts cast on the reliability of per-pixel classification which often resulted in misclassification and then a speckled appearance.

With the recent development of 'integrated' GIS, a per-parcel approach has been more and more introduced in mapping agricultural landscape. The basic idea behind this method is that agricultural field boundaries integrated with remotely sensed data divide the image into homogeneous units of image pixels, which enables pixels contained within a parcel to be processed in coherence. A parcel-based representation is most appropriate for mapping agricultural land cover by the use of crop field boundaries which can eliminate the classification errors due to the within-field spectral variability and mixed-pixels along the boundaries of fields (Dean and Smith, 2003). However, it is based on the assumption that only one crop type dominates one field, which is not always true in reality. Problems will therefore occur when mosaics of crop types distribute within one parcel structure and represent a heterogeneous landscape (Dean and Smith, 2003).

The aim of this study is to develop an integrated classifier which can switch between per-parcel and per-pixel classification to meet the accuracy requirement for both homogeneous and heterogeneous landscapes.

\section{STUDY AREA AND DATA}

\subsection{Study area}

The selected area is located at Xuzhou city, which is situated in Jiangsu Province in the southeast of China, measuring approximately $20 \mathrm{~km}^{2}\left(34^{\circ}\right.$ $\left.20^{\prime} 46^{\prime \prime} \mathrm{N}-34^{\circ} 22^{\prime} 57^{\prime \prime} \mathrm{N} ; 117^{\circ} 37^{\prime} 56^{\prime \prime} \mathrm{E}-117^{\circ} 41^{\prime} 11^{\prime \prime} \mathrm{E}\right)$ (Fig. 1). The study area is representative of the agricultural regions of the southeast level plain of China. The main crops grown in the area include rice, corn, soybean and 


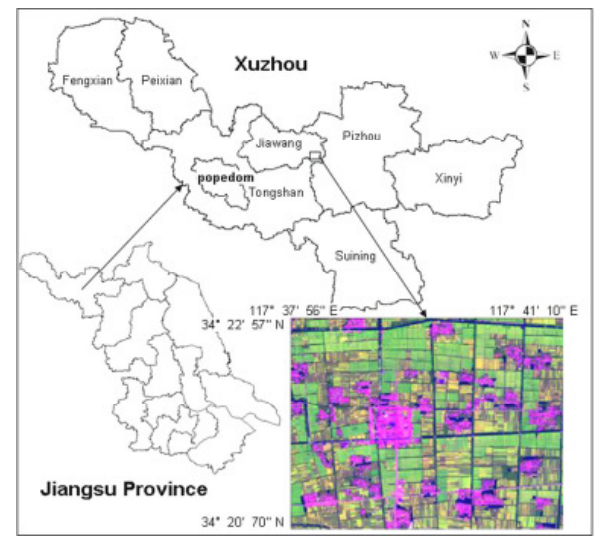

Fig. 1. The study area

mulberry. The structure of landscape is characterized by both homogeneous large rectangle rice fields and fields that are quite heterogeneous due to the irregularly scattered multiple crop types such as corn, soybean and mulberry.

\subsection{Data}

Optical satellite imagery obtained by SPOT5 HRV has been used. The optimal acquisition periods of optical satellite imagery are determined by the phonological characteristics of the main crops (Fig. 2). The SPOT5 HRV images used for classification were acquired on 17 August, 2006. All images were cloud free and of good quality. A subset $(500 \times 400$ multi-spectral $)$ and (2000×1600 panchromatic) was extracted from the full scene for the study.

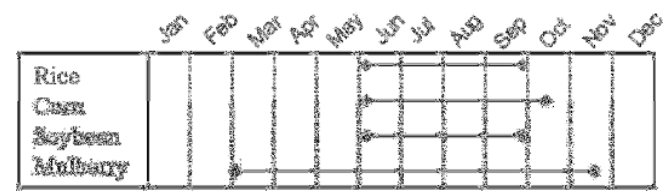

Fig. 2. The phonological characteristics of the main crops in the area

A multi-spectral image of $10-\mathrm{m}$ resolution bands and the $2.5-\mathrm{m}$ panchromatic band were fused using the Brovey algorithm to be used as a base map for vector digitizing afterwards. To ensure the accuracy of classification, the images were geometrically corrected to UTM (Zone-50) projection and datum WGS84 using 20 ground control points (GCPs). The registration errors were controlled no more than 0.5 pixels in localized areas. The vector field boundary data was delineated through manual on-screen digitalization based on the linear feature, such as ditches, roads and tree lines in the fused base map and stored as vector polygons. The SPOT5 multispectral image with the digitized vector field boundary data set overlaid is 


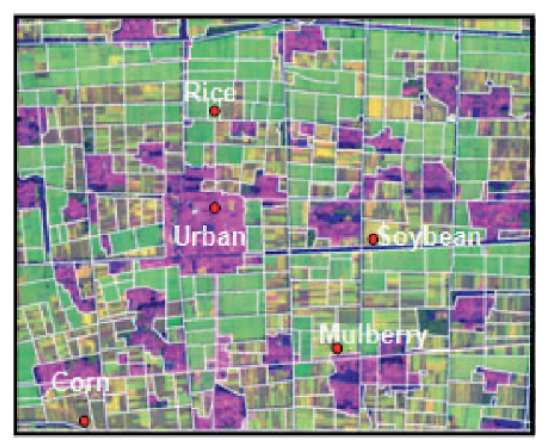

Fig. 3. A color composite of SPOT5 bands 4(SWIR). 1(green) and 2(red) with the digitized agricultural field boundaries overlaid

illustrated in Fig. 3. For crop classification only, the non-crop land cover types such as urban area, woods and water body were clipped out beforehand.

\section{METHODS}

The use of a mean spectral response for each image object in per-parcel classification against individual pixel spectral responses for each pixel in per-pixel classification was investigated in the integrated classification. In view of the main characteristics of each method, the spatial distribution of land features plays a major role in the success of classification.

A single per-parcel classification and a single per-pixel classification were respectively performed beforehand on the SPOT5 multi-spectral image. The results of each were employed as a basis and contrasts for the integrated classification. In this study, only supervised maximum likelihood classifier was used. Training sites for both per-parcel and per-pixel classification were selected from the most homogeneous image parcels by visual interpretation.

Besides classification strategies, a measure should be used to set a point at which the classifier can swift from 'per-parcel' to 'per-pixel' or versus. The selection of the measure and the determination of the point where the optimal swift happens are crucial to the integrated method. As discussed above, the intended measure should work to represent the spatial variance which is highly related to the method choice and the classification performance. Texture is just such a measure that functions in such a way that texture operators transform input image into texture coded in grey values. In practice, this study use a texture operator based on a co-occurrence matrix that measure the entropy in a $5 \times 5$ pixel window on the panchromatic band. The texture information was then incorporated into each parcel by per-parcel calculation of average textures. To determine the best swift point between per-parcel and per-pixel methods, a threshold must be denoted during the 
range of average parcel textures, above which, the results from per-parcel classification should be replaced by the per-pixel classification results to avoid the weakness of per-parcel method on heterogeneous objects. However, such a threshold can be hardly set unless an analysis operates on a gradual transition from per-parcel to per-pixel classification. The expected optimal point for method swift can then be identified.

\section{RESULTS AND DISCUSSION}

The final crop classification results from complete per-parcel classification and complete per-pixel classification are shown in Fig. 4 (a) and (b) respectively. The overgeneralization of heterogeneous parcels dominated by irregular crop mixture resulted from per-parcel classification and the speckled appearance at field boundaries and within homogeneous fields resulted from per-pixel classification can be readily recognized.
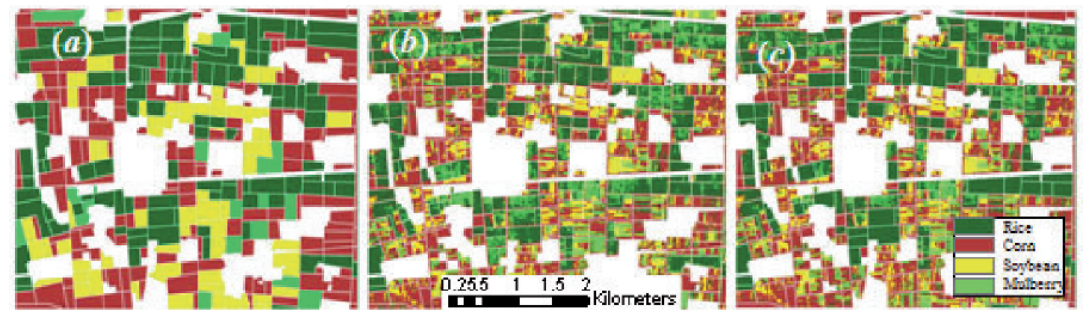

Fig. 4. (a) Crop classification map produced by per-parcel classification; (b) Crop classification map produced by per-pixel classification; (c) Crop classification map produced by an integration classification method with the optimal accuracy

For accuracy evaluation, reference data was established at a pixel level with the combination of ground data and interpretation results from the high resolution SPOT5 images. A total of 443 check points were selected for each classification results at the same locations. Two error matrices for each sole classification results are shown in Table 1 and 3. The overall, producer's and user's accuracies were calculated. A kappa index was also computed.

Table 1. Error matrix of the per-parcel crop classification

\begin{tabular}{|c|c|c|c|c|c|c|}
\hline & \multirow[b]{2}{*}{ Rice } & \multicolumn{3}{|c|}{ Reference data } & \multirow[b]{2}{*}{ Sum } & \multirow[b]{2}{*}{ U. Acc. (\%) } \\
\hline & & Corn & Soybean & Mulberry & & \\
\hline Rice & 85 & 17 & 6 & 7 & 115 & 73.91 \\
\hline Corn & 2 & 120 & 44 & 10 & 176 & 68.18 \\
\hline Soybean & 8 & 37 & 55 & 7 & 107 & 51.40 \\
\hline Mulberry & 2 & 12 & 22 & 9 & 45 & 20.00 \\
\hline Sum & 97 & 186 & 127 & 33 & 443 & \\
\hline P. Acc. $(\%)$ & 87.63 & 64.51 & 43.31 & 27.27 & & \\
\hline \multicolumn{3}{|c|}{ Overall accuracy (\%): 60.72} & \multicolumn{2}{|c|}{ Kappa index: 0.4385} & & \\
\hline
\end{tabular}

U. Acc., User's Accuracy; P. Acc., Producer's Accuracy. 
Table 2. Error matrix of the optimal integrated method of crop classification

\begin{tabular}{lcccccc}
\hline & & \multicolumn{4}{c}{ Reference data } & \\
\cline { 2 - 5 } & Rice & Corn & Soybean & Mulberry & Sum & U. Acc. (\%) \\
\hline Rice & 88 & 2 & 0 & 25 & 115 & 76.52 \\
Corn & 0 & 173 & 0 & 1 & 176 & 98.30 \\
Soybean & 5 & 5 & 92 & 5 & 107 & 85.98 \\
Mulberry & 3 & 0 & 1 & 41 & 45 & 91.11 \\
Sum & 96 & 180 & 95 & 72 & 443 & \\
P. Acc. (\%) & 91.67 & 96.11 & 96.84 & 56.94 & & \\
\multicolumn{1}{r}{ Overall accuracy (\%): 88.94} & \multicolumn{6}{c}{ Kappa index: 0.8451} \\
\hline
\end{tabular}

See Table 1 for key to abbreviations.

Table 3. Error matrix of the per-pixel crop classification

\begin{tabular}{lcccccc}
\hline & & \multicolumn{4}{c}{ Reference data } & \\
\cline { 3 - 5 } & Rice & Corn & Soybean & Mulberry & Sum & U. Acc. (\%) \\
\hline Rice & 80 & 1 & 0 & 34 & 115 & 69.57 \\
Corn & 0 & 173 & 2 & 1 & 176 & 98.30 \\
Soybean & 5 & 0 & 97 & 5 & 107 & 90.65 \\
Mulberry & 3 & 0 & 1 & 41 & 45 & 91.11 \\
Sum & 88 & 174 & 100 & 81 & 443 & \\
P. Acc. (\%) & 90.91 & 99.43 & 97.00 & 50.62 & & \\
\multicolumn{1}{c}{ Overall accuracy (\%): 88.26} & \multicolumn{6}{c}{ Kappa index: 0.8368} \\
\hline
\end{tabular}

See Table 1 for key to abbreviations.

The results from per-pixel classification appeared to be generally good compared with those from per-parcel classification. Despite the poor overall performance, results from per-parcel classification for rice were fairly good Hence, the major negative affect on the per-parcel classification resorted to those three crop types except rice. The obvious difference between rice fields and parcels of the other three crop types is their spatial variance, low in the large rectangle rice field against much high in a parcel with scattered distribution of other three crop types.

The average texture value ranged from 0 to 3.154 . The density split technique was adopted to divide the texture range equivalently into 20 subranges, and then 19 thresholds were generated (Table 4). The per-parcel classification results of those parcels whose average texture values were above the settled threshold would be replaced by correspondent results from per-pixel classification. Thus, 19 integrated classification maps with their error matrices were produced in accordance with different threshold ranges.

Table 4. Texture ranges denoted by different thresholds

\begin{tabular}{lcccccccccr}
\hline Index & 1 & 2 & 3 & 4 & 5 & 6 & 7 & 8 & 9 & 10 \\
\hline Texture & $>2.97$ & $>2.81$ & $>2.66$ & $>2.50$ & $>2.34$ & $>2.19$ & $>2.03$ & $>1.88$ & $>1.72$ & $>1.56$ \\
\hline Index & 11 & 12 & 13 & 14 & 15 & 16 & 17 & 18 & 19 & \\
\hline Texture & $>1.41$ & $>1.25$ & $>1.09$ & $>0.94$ & $>0.78$ & $>0.63$ & $>0.47$ & $>0.31$ & $>0.16$ & \\
\hline
\end{tabular}




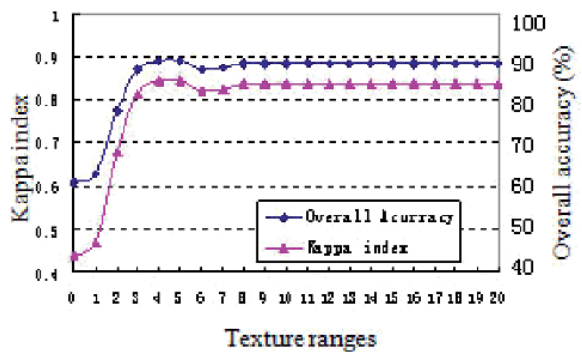

Fig. 5. Changes of overall accuracy and kappa index of classification during the transition from absolute 'parcel-based' to absolute 'pixel-based'

Notes: the numbers in the abscissa correspond to the index in Table 4. The left end ' 0 ' represents an absolute parcel-based classification while the right end '20' represents an absolute pixel-based classification

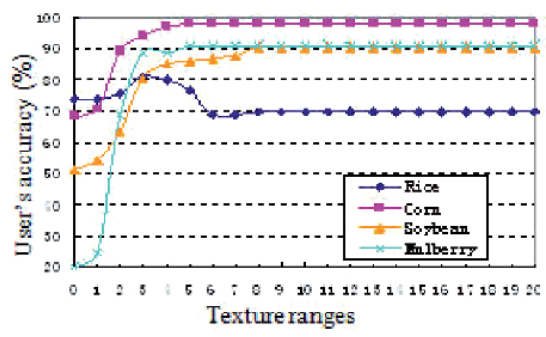

Fig. 6. Changes of user's accuracy of each crop type from absolute 'per-parcel' to absolute 'per-pixel'. See Fig. 5 for notes

The performance of these integrated classification together with that of the absolute per-parcel and per-pixel classification are organized in Fig. 5 to 7.

As shown in Fig. 5, with an increase of per-parcel classification results on heterogeneous parcels replaced by per-pixel classification results, both the overall accuracy and kappa index of the integrated classification are improved dramatically to a top level which is a little bit better than those of per-pixel classification alone. Nevertheless, the increase of accuracy is soon taken place by a decline. Afterwards, the accuracies of integrated classification retain to be almost the same as that of per-pixel classification due to an increasingly large part of per-pixel classification that shelters the advantages of per-parcel classification in processing less variable areas.

Further light was shed by examination the user's and the producer's accuracies. Fig. 6 and 7 present them by giving the explicit classification performance on each crop type, which can deduce the latent causes of the overall classification performance represented by Fig. 5. In the case of user's accuracy, all of the three crop types except rice have an obvious improvement. The only exception for rice is shown clearly by its distinct curve. As for producer's accuracy, the situation for rice was not as good as that in its user's accuracy as far as per-parcel classification was concerned to 
be compared with per-pixel classification, although the slight similarity of the curve structures in both graphs can be detected in scrutiny. Another thing worth to be mentioned in producer's accuracy is mulberry. For per-parcel classification, the comparatively small amount of mulberry distributed in the study area challenge the classification on a parcel basis at most; while for per-pixel classification, a great deal of rice pixels were misclassified to mulberry due to the spectral variability in rice fields.

An integrated classification result at the fifth texture range, the nearest one to the overall optimal choice, is given in Fig. 4 (c) and its error matrix in Table 2. The crop classification map produced by integrated classification inherited the smoothness of per-parcel classification in homogeneous parcels as well as the detail of per-pixel classification in parcels of high heterogeneity, which led to a better performance.

\section{CONCLUSION}

The integrated approach was designed to improve the accuracy of crop classification from SPOT5 HRV images data in an agricultural environment in China, typified by both homogeneous and heterogeneous crop fields. The design strategy is to combine the advantages of the per-parcel and per-pixel approaches with both spectral and spatial information. Although the optimal integrated approach represents a better performance than each of the two conventional ones to some degree, the best choice for a classification assignment largely depends on the spatial distribution of the real world. Anyway, an active analysis on the spatial characteristics of the image data is a reasonable prerequisite for any effective information extraction from remotely sensed data.

\section{REFERENCES}

De Wit A J W and Clevers J G P W. Efficiency and accuracy of per-field classification for operational crop mapping. International Journal of Remote Sensing, 2004, 25(20): 4091-4112.

Dean A M and Smith G M. An evaluation of per-parcel land cover mapping using maximum likelihood class probabilities. International Journal of Remote Sensing, 2003, 24 (14): 2905-2920.

Lo C P and Choi J. A hybrid approach to urban land use/cover mapping using Landsat 7 Enhanced Thematic Mapper Plus (ETM+) images. International Journal of Remote Sensing, 2004, 25(14): 2687-2700.

Smith G M and Fuller R M. An integrated approach to land cover classification: an example in the Island of Jersey. International Journal of Remote Sensing, 2001, 22(16): 3123-3142. 